\title{
Fluvastatin inhibits cardiomyocyte apoptosis after myocardial infarction through Toll pathway
}

\author{
LILI JIA ${ }^{1,2}$, LIANG WANG ${ }^{3}$, WENXUE LIU $^{4}$, GUANGLEI QIAN $^{4}$, XIUFANG JIANG ${ }^{5}$ and ZHIMIAN ZHANG ${ }^{6}$ \\ ${ }^{1}$ Qilu Hospital of Shandong University, Jinan, Shandong 250012; Departments of ${ }^{2}$ Nephrology, ${ }^{3}$ Cardiology, \\ ${ }^{4}$ Pediatrics and ${ }^{5}$ Obstetrics, The People's Hospital of Zhangqiu Area, Jinan, Shandong 250200; \\ ${ }^{6}$ Health Examination Center, Qilu Hospital of Shandong University, Jinan, Shandong 250012, P.R. China
}

Received December 18, 2017; Accepted June 4, 2018

DOI: $10.3892 /$ etm.2018.6297

\begin{abstract}
The present study intended to investigate the effect of fluvastatin on cardiomyocyte apoptosis after myocardial infarction in rats. Eighty myocardial infarction rat models were established and randomly divided into 4 groups $(n=20)$ : experimental group $(n=20)$ was given fluvastatin treatment; sham operation group $(n=20)$ and normal control group $(n=20)$ were given saline. The dose of fluvastatin was $20 \mathrm{mg} /(\mathrm{kg} \cdot \mathrm{d})$, and irrigation gavage was given for 1 week. Western blot analysis and reverse transcription-quantitative PCR (RT-qPCR) were used to detect the expression of TLR4 mRNA and protein in cardiomyocytes. TUNEL method was used to detect the apoptosis of cardiomyocytes. After fluvastatin treatment for 1 week, RT-qPCR found that compared with myocardial infarction group, the TLR4 mRNA expression of fluvastatin treatment group and normal control group was significantly increased, and the differences between groups were a statistically significant difference $(\mathrm{P}<0.05)$. Western blot analysis showed that compared with the myocardial infarction group, the expression of TLR4 protein in normal control group, sham operation group and fluvastatin treatment group were significantly decreased, and they all were statistically significant $(\mathrm{P}<0.05)$. TUNEL method found that compared with the myocardial infarction group, the fluvastatin treatment group could significantly reduce the apoptosis of cardiomyocytes $(19.2 \pm 3.8 \%)$, and the difference was statistically significant $(\mathrm{P}<0.05)$. Fluvastatin can inhibit myocardial infarction and decrease cardiomyocyte apoptosis by increasing the expression of TLR4-like receptor.
\end{abstract}

\section{Introduction}

Coronary atherosclerotic heart disease (CHD), also known as coronary heart disease, is caused by coronary atherosclerosis

Correspondence to: Dr Zhimian Zhang, Health Examination Center, Qilu Hospital of Shandong University, No. 107 Wenhua West Road, Jinan, Shandong 250012, P.R. China

E-mail: z9k8e5@163.com

Key words: fluvastatin, TLR4, myocardial infarction, cardiomyocyte, rat that leads to occlusion and stenosis of the coronary arteries leading to myocardial ischemia and hypoxia in patients $(1,2)$. It is the most common cardiovascular disease for the elderly, and untimely treatment will lead to disability and death, and it is also a kind of disease with high mortality rate in the elderly (3). According to statistics, there are as high as 290 million people suffering from cardiovascular diseases in China, and mortality rate is higher than $30 \%$, and the mortality and morbidity rates are now increasing year by year $(4,5)$. Acute myocardial infarction (AMI) is the most serious type with the highest incidence among coronary heart disease in the elderly, mainly caused by coronary artery stenosis due to atherosclerosis, leading to myocardial ischemia, myocardial injury, and it is the main cause of sudden death clinically. There are as many as 8.5 million patients annually who die of AMI. At present, with the aging of the population, people pay more and more attention to the health problems of the elderly. This is also one of the problems to be solved immediately (6-8).

Fluvastatin is a reductase inhibitor, which mainly reduces the synthesis and storage of cholesterol in liver cells, and also has the effect of lowering blood cholesterol and low-density lipoprotein (9). Clinically, fluvastatin is the most widely used lipid-modifying drug, which has little side-effects and adverse reactions, playing an important role in atherosclerosis (10). Tolllike receptor 4 (TLR4), an innate immune receptor, is an important member of the Toll-like receptor family. It is a class of cytokines that recognizes the early invasion of pathogens and induces cell differentiation (11). TLR4 plays an important regulatory role in wound healing, anti-infective immunity and tumor formation. The relationship between fluvastatin and TLR4 signaling pathway in cardiomyocytes of myocardial infarction (MI) rats model has not been reported in domestic and foreign literature yet. In this study, we investigated the effect of fluvastatin on cardiomyocyte apoptosis and the effect of fluvastatin on TLR4 signaling pathway by constructing MI rats, and further explored the protective effect of fluvastatin on cardiomyocytes.

\section{Materials and methods}

Experimental animals. Eighty healthy Wistar rats, male, 6-9 weeks old, weighing 180-300 g, were purchased from Beijing Vital-Lihua Experimental Animal Technology Co., Ltd. (Beijing, China). The rats were fed in separated cages at 
Table I. Primer sequences.

\begin{tabular}{lll}
\hline Gene & \multicolumn{1}{c}{ TLR4 } & \multicolumn{1}{c}{$\beta$-actin } \\
\hline Upstream & 5'-AGCAGAGGAGAAAGCATCTATGATGC-3' & 5'-AGCAGAGAATGGAAAGTCAAA-3' \\
Downstream & 5'-GGTTTAGGCCCCAGAGTTTTTCTCC-3' & 5'-ATGCTGCTTACATGTCTCGAT-3' \\
\hline
\end{tabular}

room temperature $26^{\circ} \mathrm{C}$ and regular light, with ambient noise $<45 \mathrm{~dB}$, feeding for a week in the above-mentioned environment. The study was approved by the Ethics Committee of Qilu Hospital of Shandong University (Jinan, China).

Experimental model construction and grouping. Eighty rats were randomly divided into normal control group $(n=20)$, sham operation group $(n=20)$, IM group $(n=20)$ and fluvastatin treatment group $(n=20)$. Rat IM model was established: Rats were anesthetized with $10 \%$ chloral hydrate $(300 \mu \mathrm{l} / \mathrm{g})$; tracheal intubation was used for assisted respiration in rats; the thoracic and pericardium were opened by surgery to expose the heart; the needle was inserted at the left atrial appendage; the coronary artery was ligated below the pulmonary cone by using 5/0 thread. The anterior part of the local ventricle was white after the ligation; while in the sham operation group, the threading was not ligated. After operation, fluvastatin treatment group was given intragastric administration of $[30 \mathrm{mg} /(\mathrm{kg} \cdot \mathrm{d})]$, and the other groups were given intragastric administration of an equal volume of normal saline, each group received gavage for 1 week.

Sample processing. On day 7 of lavage, 10\% KCI (3 ml) was injected through the jugular vein. When the heart stopped beating, the chest was opened immediately and the heart was cut, placed in saline for cleaning. The left and right atrial appendage and blood vessels were removed and the heart was dried with filter paper; the heart was cut into two parts in the papillary muscle plane; $200 \mathrm{mg}$ tissue was obtained near the apical non-infarcted area for the determination of TLR4 protein; the other part was placed in a $-80^{\circ} \mathrm{C}$ refrigerator for preservation.

Cardiomyocyte apoptosis detection. TUNEL assay kit was used to detect the myocardial apoptotic cells in each group, and the operation was performed strictly according to the instructions of the kit. Apoptotic cardiomyocytes nuclei stained yellow or yellow-brown, and normal myocardial nuclei stained blue. The cells were counted using light microscope (BX-42; Olympus, Tokyo, Japan). Five fields were randomly selected for each slide, and the apoptotic cells in the field of vision were counted. The apoptosis rate $(\mathrm{AI})=$ total apoptosis/total cell number x $100 \%$.

Western blot method. The spare cells were extracted and added into the cell lysis solution. The RIPA lysis buffer (Sigma-Aldrich, St. Louis, MO, USA) was placed on the ice for $1 \mathrm{~h}$. After shaking vigorously for 1-2 times every $10 \mathrm{~min}$, the cells were centrifuged at $9,350 \mathrm{x}$ for $10 \mathrm{~min}$ at $4^{\circ} \mathrm{C}$. The supernatant was taken and the protein concentration was measured by the BCA method. A total of $30 \mu \mathrm{g}$ of total protein was extracted and subjected to a buffer. After denaturation at $94^{\circ} \mathrm{C}$ for $3 \mathrm{~min}$, the protein was separated by $12 \%$ SDS-PAGE electrophoresis and transferred to a PVDF membrane at a constant current of $350 \mathrm{~mA}$. The membrane was placed in TBS buffer with 5\% skim milk in room temperature and in the dark for blocking for $1 \mathrm{~h}$, and placed overnight at $4^{\circ} \mathrm{C}$ with the corresponding antibody; rinsed 3 times with TBS buffer on the next day for 5-10 min each, goat anti-rabbit and goat anti-rat IgG were marked with HRP $(1: 2,000)$, then washed with TBS buffer three times for 5-10 min each, fixed and developed by using a color-developing system, and light-emitting imaging technology was used for exposure imaging and grayscale scanning.

Reverse transcription-qPCR (RT-qPCR) detection of tissue $T L R 4$. The spare tissue was added with TRIzol reagent, shook and placed at room temperature for $30 \mathrm{~min}$ for complete lysis. Total RNA was extracted from each group of cells according to the kit manual, and the extraction process was performed strictly according to the instructions. The extracted RNA was analyzed by UV spectrophotometer (Hitachi, Tokyo, Japan) and protein electrophoresis to determine its concentration and purity. The extracted total RNA was reverse-transcribed according to the instructions of the reverse transcription kit. The extracted cDNA samples were stored at $-20^{\circ} \mathrm{C}$. TLR4 primers designed by Shanghai Sangon Biological Engineering Corp., (Shanghai, Chian) are shown in Table I. PCR reaction system was designed according to the instructions, and the total system was $12.62 \mu \mathrm{l}$, using DEPC to make up to $20 \mu \mathrm{l}$. PCR reaction conditions: $94^{\circ} \mathrm{C}$ pre-denaturation $10 \mathrm{~min}$; $94^{\circ} \mathrm{C} 45 \mathrm{sec}, 60^{\circ} \mathrm{C} 45 \mathrm{sec}, 72^{\circ} \mathrm{C} 45 \mathrm{sec}$, a total of 40 cycles. The manufacturer's software was used for amplification data analysis, and $\beta$-actin was used as an internal reference gene.

Statistical analysis. SPSS 19.0 software (SPSS, Inc., Chicago, IL, USA) was used for statistical analysis. The data were expressed as mean $\pm \mathrm{SD}$, and the mean value of multiple groups were compared by one-way ANOVA. Comparison between each two groups were by Student-Newman-Keuls (SNK) q-test or LSD-t. $\mathrm{P}<0.05$ was considered statistically significant.

\section{Results}

Modeling results. In this experiment, among a total of 80 rats, 60 were performed with coronary artery ligation, and 38 survived 4 days after the operation; the other 20 rats were normal control group.

$R T-q P C R$ detection of the expression of TLR4 mRNA in the cells. In this experiment, we detected the expression of TLR4 mRNA by RT-qPCR in rat cardiomyocytes of four groups, 


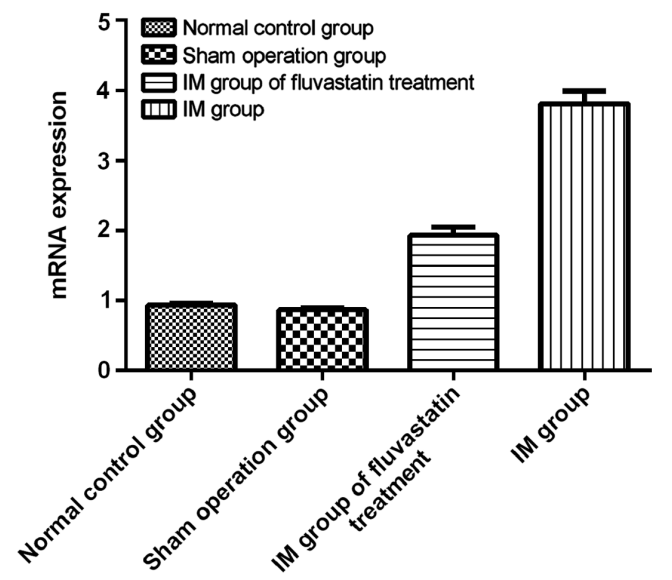

Figure 1. Expressions of TLR4 mRNA in each group. There was significant difference $(\mathrm{P}<0.05)$ in terms of the expression between each group according to reverse transcription-qPCR; there was difference between the fluvastatin treatment group and the sham operation group, the normal group $(\mathrm{P}<0.05)$; there was no difference between sham operation group and normal control group $(\mathrm{P}>0.05)$.

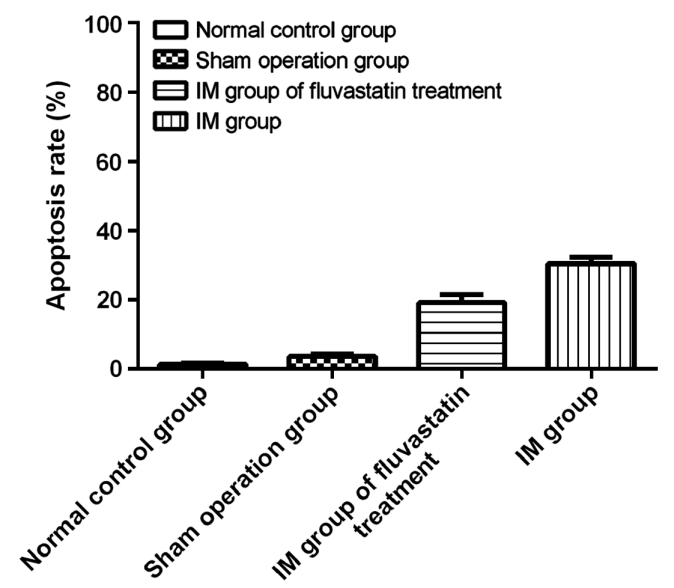

Figure 2. Apoptosis of cardiomyocytes in each group by TUNEL method. There was significant difference in apoptosis of cardiomyocytes between the sham operation group and the normal group $(\mathrm{P}>0.05)$, there was difference between the IM group and the sham operation group $(\mathrm{P}<0.05)$, there was difference between the IM group and the normal group $(\mathrm{P}<0.05)$, there was difference between the fluvastatin treatment group and the sham operation group $(\mathrm{P}<0.05)$, there was difference between the fluvastatin treatment group and the normal group $(\mathrm{P}<0.05)$, there was difference between the IM group and the fluvastatin treatment group $(\mathrm{P}<0.05)$.

and the results showed that TLR4 mRNA was expressed in all four groups. The expression of TLR4 in the normal control group was $0.902 \pm 0.140$ and the TLR4 expression in the sham operation group was $1.214 \pm 0.274$. TLR4 expression in IM group was $3.412 \pm 0.879$ and TLR4 expression in fluvastatin treatment group was $1.864 \pm 0.467$. Compared with IM group, TLR4 mRNA expression in sham operation group, fluvastatin treatment group and normal control group were significantly increased, and comparison in each group was statistically significant $(\mathrm{P}<0.05)$, indicating that fluvastatin can reduce the expression of TLR4mRNA in cardiomyocytes. There was statistical significance between fluvastatin treatment group and sham operation group and normal control group $(\mathrm{P}<0.05)$; there was no statistical difference between normal
Table II. Protein and mRNA expression.

\begin{tabular}{lcll}
\hline Group & No. & TLR4 mRNA & TLR4 protein \\
\hline Normal control & 20 & $0.902 \pm 0.140^{\mathrm{a}}$ & $0.384 \pm 0.034^{\mathrm{a}}$ \\
Sham operation & 20 & $1.214 \pm 0.274^{\mathrm{a}, \mathrm{b}}$ & $0.391 \pm 0.048^{\mathrm{a}, \mathrm{b}}$ \\
Fluvastatin treatment & 20 & $1.864 \pm 0.467^{\mathrm{a}-\mathrm{c}}$ & $0.423 \pm 0.074^{\mathrm{a}-\mathrm{c}}$ \\
IM & 20 & $3.412 \pm 0.879$ & $0.574 \pm 0.098$
\end{tabular}

${ }^{\mathrm{a}} \mathrm{P}<0.05$ compared with the IM group; ${ }^{\mathrm{b}} \mathrm{P}<0.05$ compared with the normal control group; ${ }^{\mathrm{C}} \mathrm{P}<0.05$ compared with the sham operation group.

control group and sham operation group $(\mathrm{P}>0.05)$ (Table II and Fig. 1).

Western blot analysis. In this experiment, the expression of TLR4 protein in rat cardiomyocytes was detected by western blot analysis. It was observed that TLR4 protein was expressed in all four groups. Compared with IM group, the expression of TLR4 protein in normal control group, sham operation group and fluvastatin treatment group were all significantly decreased, and the differences were statistically significant $(\mathrm{P}<0.05)$. The difference between the normal control group and the fluvastatin group, sham operation group was statistically significant $(\mathrm{P}<0.05)$, and there was no significant difference between the normal control group and the sham operation group $(\mathrm{P}>0.05)$ (Table II).

Cardiomyocyte apoptosis. In this experiment, the apoptosis of rat cardiomyocytes was detected by TUNEL method. The nuclei of TUNEL-positive apoptotic cells were brownish yellow and the nuclei of TUNEL-negative was blue. It was observed that in normal control group, no apoptotic cardiomyocytes were found; a very small amount of cardiomyocytes in sham operation group occurred apoptosis; the amount of apoptosis cells in IM group increased significantly compared with normal control group and sham operation group (30.4 $\pm 3.1 \%)$, and the difference was statistically significant; compared with the IM group, fluvastatin treatment could significantly reduce the apoptosis of cardiomyocytes (19.2 $\pm 3.8 \%)$, and the difference was statistically significant $(\mathrm{P}<0.05)$; there was no difference between normal control group and sham operation group $(\mathrm{P}>0.05)$ (Fig. 2).

\section{Discussion}

Apoptosis, also known as apoptosis, is a form of active death that occurs when genes are regulating cells. It plays a very important role in the growth and development of normal tissues and in the development of diseases $(12,13)$. There are many factors that can lead to apoptosis of cells. There are studies which have shown that the main factors of apoptosis were hypoxia, persistent ischemia and reperfusion (14). It has been reported (15) that ischemia-induced perfusion injury in rabbit models leads to apoptosis in rabbit cardiomyocytes, and other studies (16) have shown that cardiomyocytes apoptosis was found in the myocardium of patients with acute IM and 
end-stage heart failure. Cell apoptosis is directly controlled by genes that control apoptosis, and extracellular signals regulate cells and eventually cause cell death. There are many reasons for the apoptosis of myocardial cells after IM such as neurohormone system, inflammatory cytokines and CO (17). Because of the coexistence of multiple factors, it is very difficult to distinguish the role played by IM in a certain period of time and one stimulus. We found through animal experiments that after myocardial infarction, myocardial apoptosis occurs in rats at different time-points after IM, and cardiomyocyte apoptosis is mediated by multiple death signals and regulated by different genes. The mechanism of different genes on myocardial apoptosis is not yet fully understood.

TLR4, as a natural immune receptor, its mRNA expression was found for the first time in animal models of cardiomyocytes, and Kim et al (18) pointed out that activation of TLR4 signaling pathway can induce cardiomyocyte apoptosis, and the use of TLR4 blocking antibody to block TLR4 eventually leads to diminished cardiomyocyte apoptosis. It has been reported (19) that after TLR4 activation after acute IM, a large number of inflammatory factors are expressed. The release of inflammatory cytokines is related to cardiomyocyte apoptosis, and TLR4 signaling pathway-mediated apoptosis may be related to the release of inflammatory cytokines under TLR4-mediated conditions (20). Fluvastatin, as the first HMG-CoA reduction inhibitor, has obvious difference from other statins and its structure is a special open-ring type. Fluvastatin can produce better pharmacological activity without metabolic transformation; in addition to lipid-lowering function, it also has the functions of stabling plaque, suppressing inflammation and regulating coagulation (21).

In the present study, we explored the role of fluvastatin in myocardial cells of IM rats by treating rat IM model with fluvastatin and activating TLR4 signaling pathway to establish a model. The expression of TLR4 in cardiomyocytes of 4 groups was detected by TUNEL, western blot analysis and RT-qPCR. The results showed that the expression of TLR4 mRNA in all 4 groups of cardiomyocytes was detected by RT-qPCR, and was significantly expressed in IM group, which is consistent with the study of Yang et al (22): The expression of TLR4 mRNA in cardiomyocytes was significantly increased after IM. Liu et al (23) have found that after long-term IM, myocardial cells TLR4 and pro-inflammatory cytokines are upregulated to promote inflammation and thus increase heart failure. TLR4 expression in normal control and sham operation group was significantly different from that in IM group, while TLR4 expression in myocardial cells treated with fluvastatin decreased significantly compared with IM group. The expression of TLR 4 protein in 4 groups of rats was detected by western blot, and the results showed that TLR4 protein expression in IM group was significantly higher than that in other groups, which was consistent with the conclusion of Sun et al (24). It is suggested that fluvastatin can inhibit the expression of TLR4 to reduce the apoptosis of cardiomyocytes, thus we detected the apoptosis of cardiomyocytes by TUNEL method. It was observed that there was a significant difference in the apoptosis of rat cardiomyocytes between the fluvastatin treatment group and the IM group $(19.2 \pm 3.8$ vs. $30.4 \pm 3.1 \%$, $\mathrm{P}<0.05)$, indicating that fluvastatin can regulate cardiomyocyte apoptosis by inhibiting the expression of TLR4.
This experiment showed that fluvastatin can inhibit the expression of TLR4, however, the specific mechanism of TLR4 inhibition is unclear, whether fluvastatin modulates TLR4 directly or indirectly through other proteins needs further study and experiments, and this experiment was based on the animal model, and whether different modeling methods and practices will affect the experimental results also needs to be studied in depth in the future.

In summary, fluvastatin can decrease the apoptosis of cardiomyocytes and inhibit IM by increasing the expression of TLR4-like receptors.

\section{Acknowledgements}

Not applicable.

\section{Funding}

No funding was received,

\section{Availability of data and materials}

The datasets used and/or analyzed during the present study are available from the corresponding author on reasonable request.

\section{Authors' contributions}

LJ drafted the manuscript. LJ and $\mathrm{ZZ}$ were mainly devoted to collecting and interpreting the data. WL, LW and GQ helped with RT-qPCR. XJ and ZZ were responsible for statistical analysis. All authors have read and approved the final study.

\section{Ethics approval and consent to participate}

The study was approved by the Ethics Committee of Qilu Hospital of Shandong University (Jinan, China).

\section{Patient consent for publication}

Not applicable.

\section{Competing interests}

The authors declare that they have no competing interests.

\section{References}

1. Thiene G, Corrado D and Basso C: Sudden cardiac death in the young and athletes. Springer Milan 2016: 21-71, 2016.

2. Sankar NM, Ramani SS and Anantharaman R: Coronary artery disease in women. Indian Heart J 69: 807-808, 2017.

3. Forman DE, Alexander K, Brindis RG, Curtis AB, Maurer M, Rich MW, Sperling L and Wenger NK: Improved cardiovascular disease outcomes in older adults. F1000Res 5: 1-9, 2016.

4. Zhou M, Wang H, Zhu J, Chen W, Wang L, Liu S, Li Y, Wang L, Liu Y, Yin P, et al: Cause-specific mortality for 240 causes in China during 1990-2013: A systematic subnational analysis for the Global Burden of Disease Study 2013. Lancet 387: 251-272, 2016.

5. Chan NY: Sudden cardiac death in Asia and China: Are we different? J Am Coll Cardiol 67: 590-592, 2016.

6. Reed GW, Rossi JE and Cannon CP: Acute myocardial infarction. Lancet 389: 197-210, 2017.

7. Stewart WJ: Atrial myocardial infarction: A neglected stalker in coronary patients. J Am Coll Cardiol 70: 2890-2892, 2017. 
8. Li S, Guo LZ, Kim MH, Han JY and Serebruany V: Platelet microRNA for predicting acute myocardial infarction. J Thromb Thrombolysis 44: 556-564, 2017.

9. Kataoka Y, Andrews J, Puri R, Psaltis P and Nicholls SJ: Lipid lowering therapy to modify plaque microstructures. J Atheroscler Thromb 24: 360-372, 2017.

10. Ruel I, Aljenedil S, Sadri I, de Varennes É, Hegele RA, Couture P, Bergeron J, Wanneh E, Baass A, Dufour R, et al: Imputation of baseline LDL cholesterol concentration in patients with familia hypercholesterolemia on statins or ezetimibe. Clin Chem 70 2022, 2017.

11. Zhai Y, Ao L, Cleveland JC, Zeng Q, Reece TB, Fullerton DA and Meng X: Toll-like receptor 4 mediates the inflammatory responses and matrix protein remodeling in remote non-ischemic myocardium in a mouse model of myocardial ischemia and reperfusion. PLoS One 10: e0121853, 2015.

12. Czabotar PE, Lessene G, Strasser A and Adams JM: Control of apoptosis by the BCL-2 protein family: Implications for physiology and therapy. Nat Rev Mol Cell Biol 15: 49-63, 2014.

13. Wang X, Sun Y, Yang H, Lu Y and Li L: Oxidized low-density lipoprotein induces apoptosis in cultured neonatal rat cardiomyocytes by modulating the TLR4/NF- $\mathrm{B}$ pathway. Sci Rep 6: 27866, 2016.

14. Tixeira R, Caruso S, Paone S, Baxter AA, Atkin-Smith GK, Hulett MD and Poon IK: Defining the morphologic features and products of cell disassembly during apoptosis. Apoptosis 22: 475-477, 2017

15. Chen Z, Wu Z, Huang C, Zhao Y, Zhou Y, Zhou X, Lu X, Mao L and Li S: Effect of lipoxin A4 on myocardial ischemia reperfusion injury following cardiac arrest in a rabbit model. Inflammation 36: 468-475, 2013.

16. Kleinbongard P, Schulz R and Heusch G: TNFa in myocardial ischemia/reperfusion, remodeling and heart failure. Heart Fail Rev 16: 49-69, 2011.

17. Liu Q, Zhang J, Xu Y, Huang Y and Wu C: Effect of carvedilol on cardiomyocyte apoptosis in a rat model of myocardial infarction: A role for toll-like receptor 4. Indian J Pharmacol 45: 458-463, 2013.
18. Kim SC, Stice JP, Chen L, Jung JS, Gupta S, Wang Y, Baumgarten G, Trial J and Knowlton AA: Extracellular heat shock protein 60 , cardiac myocytes, and apoptosis. Circ Res 105: 1186-1195, 2009.

19. Satoh M, Shimoda Y, Maesawa C, Akatsu T, Ishikawa Y, Minami Y, Hiramori K and Nakamura M: Activated toll-like receptor 4 in monocytes is associated with heart failure after acute myocardial infarction. Int J Cardiol 109: 226-234, 2006.

20. Zhai Y, Shen XD, O'Connell R, Gao F, Lassman C, Busuttil RW, Cheng G and Kupiec-Weglinski JW: Cutting edge: TLR4 activation mediates liver ischemia/reperfusion inflammatory response via IFN regulatory factor 3-dependent MyD88-independent pathway. J Immunol 173: 7115-7119, 2004.

21. Li Z, Wang L, Hu X, Zhang P, Chen Y, Liu X, Xu M, Zhang Y and Zhang M: Effect of rosuvastatin on atherosclerotic plaque stability: An intravascular ultrasound elastography study. Atherosclerosis 248: 27-35, 2016

22. Yang J, Yang J, Ding JW, Chen LH, Wang YL, Li S and Wu H: Sequential expression of TLR4 and its effects on the myocardium of rats with myocardial ischemia-reperfusion injury. Inflammation 31: 304-312, 2008.

23. Liu L, Wang Y, Cao ZY, Wang MM, Liu XM, Gao T, Hu QK, Yuan WJ and Lin L: Up-regulated TLR4 in cardiomyocytes exacerbates heart failure after long-term myocardial infarction. J Cell Mol Med 19: 2728-2740, 2015.

24. Sun Y, Huang $J$ and Song K: BET protein inhibition mitigates acute myocardial infarction damage in rats via the TLR4/TRAF6/NF-кB pathway. Exp Ther Med 10: 2319-2324, 2015. International (CC BY-NC-ND 4.0) License. 